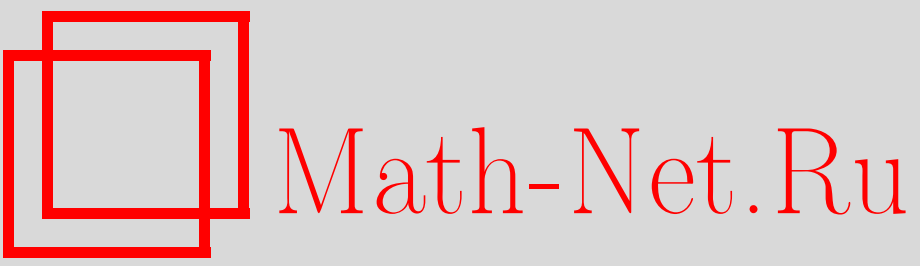

В. В. Галатенко, Т. П. Лукашенко, В. А. Садовничий, Орторекурсивные разложения и их свойства, Итоги науки и техн. Сер. Соврем. мат. и ее прил. Tемат. обз., 2019, том 170, 62-70

DOI: https://doi.org/10.36535/0233-6723-2019-170-62-70

Использование Общероссийского математического портала Math-Net.Ru подразумевает, что вы прочитали и согласны с пользовательским соглашением

http: //www.mathnet.ru/rus/agreement

Параметры загрузки:

IP: 18.208 .226 .222

26 апреля 2023 г., $17: 13: 42$ 


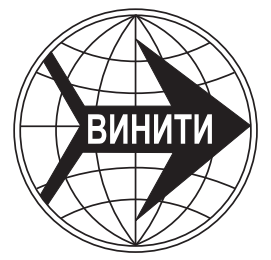

ИТОГИ НАУКИ И ТЕХНИКИ.

Современная математика и ее приложения.

Тематические обзоры.

Том 170 (2019). С. $62-70$

DOI: $10.36535 / 0233-6723-2019-170-62-70$

УДК 517.51, 517.98

\title{
ОРТОРЕКУРСИВНЫЕ РАЗЛОЖЕНИЯ И ИХ СВОЙСТВА
}

\author{
(c) 2019 г \\ В. В. ГАЛАТЕНКО, Т. П. ЛУКАШЕНКО, В. А. САДОВНИЧИЙ
}

АннотАция. Статья посвящена введеным в 1999 г. орторекурсивным разложениям - обобщениям ортогональных разложений. В ней изложены полученные за 20 лет основные результаты о свойствах орторекурсивных разложений и указаны некоторые направления дальнейших исследований.

Ключевые слова: орторекурсивное разложение, ортогональная система, фрейм, разложение, сходимость.

\section{ORTHORECURSIVE DECOMPOSITIONS AND THEIR PROPERTIES}

\author{
(c) 2019 V. V. GAlATENKO, T. P. LUKASHENKO, V. A. SADOVNICHII
}

\begin{abstract}
This paper is devoted to orthorecursive decompositions introduced in 1999, which generalize the notion of orthogonal decompositions. We present main results obtained over the last 20 years about properties of orthorecursive decompositions and indicate some areas for further research.
\end{abstract}

Keywords and phrases: orthorecursive decomposition, orthogonal system, frame, decomposition, convergence.

AMS Subject Classification: 06D22, 43Axx

Понятие орторекурсивного разложения было введено Т. П. Лукашенко в 1999 г. на симпозиуме по рядам Фурье (см. [22]) и на конференции по теории функций (см. [23]); подробная публикация [24] вышла в 2001 г. Приведем определение.

Пусть $H$ - гильбертово пространство над полем $\mathbb{R}$ или $\mathbb{C}$, а $\left\{e_{k}\right\}$ - конечная или счетная система ненулевых элементов $H$, последовательно занумерованная натуральными числами $1, \ldots, K$ или всеми натуральными числами.

Определение 1. Орторекурсивное разложение (ОРР) элемента $f \in H$ по последовательности элементов $\left\{e_{k}\right\}$ осуществляется следующим образом:

(1) положим $r_{0}=f$;

(2) если задан остаток приближения $r_{n-1} \in H, n \in \mathbb{N}$, и элемент $e_{n}$, то полагаем

$$
\hat{f}_{n}=\left(r_{n-1}, e_{n}\right)\left\|e_{n+1}\right\|^{-2}, \quad r_{n}=r_{n-1}-\hat{f}_{n} e_{n} .
$$

Назовем $\hat{f}_{k}$ рекурсивными коэффициентами Фуръе элемента $f \in H$ по системе $\left\{e_{k}\right\}$, а ряд $\sum_{k} \hat{f}_{k} e_{k}-$ рекурсивным рядом Фурье элемента $f \in H$ по системе $\left\{e_{k}\right\}$.

Легко видеть, что

$$
r_{n}(f)=f-\sum_{k \leqslant n} \hat{f}_{k} e_{k}
$$

и для ортогональной системы функций $\left\{e_{k}\right\}$ рекурсивные коэффициенты Фурье являются обычными коэффициентами Фурье, а рекурсивный ряд Фурье - обычным рядом Фурье. Из (1) следует 
равенство Пифагора

$$
\left\|r_{n-1}\right\|^{2}=\left\|r_{n}\right\|^{2}+\left|\hat{f}_{n}\right|^{2}\left\|e_{n}\right\|^{2} .
$$

Вариантом равенства $(2)$ является следующее равенство. Если остатки приближения $r_{n}(f)$ и $r_{n}(g)$ элементов $f$ и $g$ соответственно получаются на $n$-м шагу из остатков приближения $r_{n-1}(f)$ и $r_{n-1}(g)$ с использованием одного и того же элемента $e_{n}$, то

$$
\left(r_{n-1}(f), r_{n-1}(f)\right)=\left(r_{n}(f), r_{n}(g)\right)+\hat{f}_{n} \overline{\hat{g}}_{n}\left\|e_{n}\right\|^{2},
$$

где черта означает комплексное сопряжение.

Суммируя по $n$ равенство (2), получим аналог тождества Бесселя

$$
\|f\|^{2}=\sum_{n=1}^{N}\left|\hat{f}_{n}\right|^{2}\left\|e_{n}\right\|^{2}+\left\|r_{N}(f)\right\|^{2}=\left\|f-S_{N}(f)\right\|^{2}+\sum_{n=1}^{N}\left|\hat{f}_{n}\right|^{2}\left\|e_{n}\right\|^{2},
$$

а суммируя по $n$ равенство (3), получим его вариант

$$
(f, g)=\sum_{n=1}^{N} \hat{f}_{n} \overline{\hat{g}}_{n}\left\|e_{n}\right\|^{2}+\left(r_{N}(f), r_{N}(g)\right) .
$$

Из (4) следует аналог неравенства Бесселя

$$
\|f\|^{2} \geqslant \sum_{n}\left|\hat{f}_{n}\right|^{2}\left\|e_{n}\right\|^{2}
$$

и утверждение, что равенство

$$
f=\sum_{n} \hat{f}_{n} e_{n}
$$

имеет место тогда и только тогда, когда выполняется аналог равенства Парсеваля

$$
\|f\|^{2}=\sum_{n}\left|\hat{f}_{n}\right|^{2}\left\|e_{n}\right\|^{2} .
$$

Из (5) следует верность следующего аналога равенства Парсеваля: если

$$
f=\sum_{n} \hat{f}_{n} e_{n} \quad \text { или } \quad g=\sum_{n} \hat{g}_{n} e_{n},
$$

TO

$$
(f, g)=\sum_{n} \hat{f}_{n} \overline{\hat{g}_{n}}\left\|e_{n}\right\|^{2} .
$$

Отметим, что возможны две существенно различающиеся схемы орторекурсивных разложений.

1. Можно после получения остатка $r_{n-1}$ выбирать следующий элемент $e_{n}$ в зависимости от достигнутого результата (т.е. в зависимости от предыдущих шагов и остатка $r_{m-1}$ ). Этот подход реализован в появившихся раньше так называемых жадных алгоритмах (greedy algorithms; см., например, [?]).

2. Можно фиксировать систему элементов $\left\{e_{n}\right\}$ и их порядок для всего процесса разложения.

В первой схеме имеется возможность оптимизации (в различных смыслах) процесса разложения, но отсутствует линейность разложения. Вторая схема привлекательна линейностью разложения и отсутствием усложняющего разложение алгоритма оптимизации (выбора следующего элемента). Далее в статье рассматривается относящиеся только к этой схеме результаты.

В конце XX в. большой интерес вызвали всплески (вейвлеты) - системы функций, порождаемые сжатиями и сдвигами одной функции. Такие системы являются удобным аппаратом как в теоретических, так и особенно в прикладных исследованиях при цифровой обработке сигналов (см. $[1,10,39,41,52,54,59])$.

Пусть $\varphi$ - действительно- или комплекснозначная функций на вещественной прямой из пространства Лебега $L^{2}(\mathbb{R})$. Функции

$$
\varphi_{k, l}(x)=2^{k / 2} \varphi\left(2^{k} x-l\right), \quad k, l \in \mathbb{Z}, \quad x \in \mathbb{R},
$$

- система всплесков, порожденная функцией $\varphi$. 
Обычно стараются добиться, чтобы это была ортогональная система (или фрейм), при этом порождающая функция часто задается сложным выражением (например, всплески Добеши, Мейера и др.). При орторекурсивном разложении такого требования нет, и порождающая функция может быть выбрана достаточно произвольно. А. Ю. Кудрявцев исследовал условия, при которых орторекурсивное разложение сходится к разлагаемой функции (см. [12-21]). Приведем два его результата.

Теорема 1. Пусть функиия $\varphi \in L^{2}(\mathbb{R})$ и удовлетворяет следующим условиям:

(0) $\int_{0}^{1}\left(\sum_{m \in \mathbb{Z}}|\varphi(x+m)|\right)^{2} d x<\infty$

(1) $\int_{\mathbb{R}} \varphi(x) d x \neq 0$

(2) существует такая невозрастающая на промежутке $[0,+\infty)$ функиия $F(w)$, что

$$
|\varphi(w)| \leqslant F(|w|) \quad \forall w \in \mathbb{R}, \quad \int_{0}^{\infty} F^{2}(w) \ln (1+w) d w<\infty .
$$

Тогда найдется такая последовательность цельх неотрицательных чисел $L=\left\{L_{k}\right\}_{k=0}^{+\infty}$, что система $\Phi_{+}(L)=\left\{\varphi_{k, l}: k \geqslant 0,|l| \leqslant L_{k}\right\}$, а также любая ее подсистема, содержащая бесконечное число пачек (по $k)$, является безусловной относительно перестановок пачек орторекурсивной системой разложения в $L^{2}(\mathbb{R})$.

Им также указано, какими в зависимости от модуля непрерывности, можно брать числа $L_{k}$. Следующая теорема показывает, что без ограничений на функцию $\varphi$ орторекурсивное разложение может расходится.

Теорема 2. Существует такая функиия $\varphi \in L^{2}(\mathbb{R})$ с ортогональными целочисленными сдвигами, преобразование Фурье которой удовлетворяет условию $\inf _{|w|<1 / 2}|\hat{\varphi}(w)|>0$, что орторекурсивное разложение некоторой функиии $f \in L^{2}(\mathbb{R})$ по системе $\Phi_{+}=\left\{\varphi_{k, l}: k \geqslant 0, l \in \mathbb{Z}\right\}$ расходится в пространстве $L^{2}(\mathbb{R})$.

А. Ю. Кудрявцев также показал, что для любой функции $\varphi$ можно выбрать подпоследовательность пачек так, что орторекурсивное разложение любой функции из $L^{2}(\mathbb{R})$ будет сходится к ней. Он же получил оценки скорости сходимости орторекурсивных разложений по неортогональным всплескам.

А. В. Политов в других терминах изучал сходимость по системе всплесков, порожденных функцией $\varphi$ (см. [42-50].

Теорема 3. Пусть

$$
\varphi \in L^{2}[0,1), \quad \int_{0}^{1} \varphi(x) d x \neq 0, \quad \sum_{k=0}^{\infty} w_{2}^{2}\left(\varphi, 2^{-k}\right)<\infty,
$$

где $w_{2}(\varphi, \delta)$ - интегральный модуль непрерывности в $L^{2}[0,1)$. Тогда для любого элемента из $L^{2}[0,1)$ орторекурсивное разложение этого элемента по системе двоичных сжсатий и сдвигов функиии ч сходится к нему.

Для действительного гильбертова пространства А. В. Политов установил критерий сходимости орторекурсивных разложений в терминах матрицы Грама. Пусть $\mathcal{E}=\left\{e_{k}\right\}_{k=1}^{\infty}$-счетная нормированная система элементов гильбертова пространства $H$. Обозначим через $G$ матрицу Грама системы $\mathcal{E}$, т.е. матрицу, состоящую из скалярных произведений $g_{i, j}=\left(e_{i}, e_{j}\right)$, а через $Q$ - треугольную матрицу, у которой элементы, лежащие не выше главной диагонали, совпадают с элементами $G$, а остальные равны нулю. Для произвольного $e_{n} \in \mathcal{E}$ рассмотрим его орторекурсивный 
ряд по системе $\mathcal{E}$, который имеет вид $\sum_{k=1}^{\infty} c_{n, k} e_{k}$, где $c_{n, k}=\widehat{\left(e_{n}\right)_{k}}$. Матрицу, состоящую из чисел $c_{n, k} k$, обозначим $C$.

Теорема 4. Пусть замыкание линейной оболочки системы $\mathcal{E}$ совпадает с $H$. Следующие утверждения эквивалентны.

(1) Орторекурсивное разложение любого элемента $f \in H$ по системе $\mathcal{E}$ сходится $\kappa f$;

(2) выполнена система условий:

(a) $G\left(Q^{-1} G\right)=G$,

(b) $(\mathrm{CH}) \mathrm{C}^{\top}=C\left(H \mathrm{C}^{\top}\right)$, где $C^{\top}-$ транспонированная матрица $C$.

Другой класс систем, по которым изучались орторекурсивные разложения, - характеристические функции некоторых множеств. Так, в $[23,24]$ рассматривалась система $\Xi=\left\{\Delta_{k}\right\}_{k \in \mathbb{N}}$ конечных невырожденных промежутков, принадлежащих промежутку $I \subset \mathbb{R}, \chi_{k}$-характеристическая функция $\Delta_{k}, k \in \mathbb{N}$. Доказана следующая теорема.

Теорема 5. Пусть промежутки системы $\Xi=\left\{\Delta_{k}\right\}_{k \in \mathbb{N}}$ покрывают I в смысле Витали (т.е. для любых $x \in I$ u $\varepsilon>0$ найдется такой промежуток $\Delta_{k} \ni x$, что его длина $\left.\left|\Delta_{k}\right|<\varepsilon\right)$ и если $\Delta_{i} \cap \Delta_{j} \neq \varnothing$ и $i<j$, то $\Delta_{i} \supset \Delta_{j}$. Тогда для любой функиии $f$, интегрируемой в смъсле Лебега (или даже Данжуа-Перрона (Куривейля-Хенстока), см. $[38,40,51])$ на всех промежутках $\Delta_{k}$ системы $\Xi$, частичная сумма орторекурсивного ряда Фурье $\sum_{k} \hat{f}_{k} \chi_{k}$ по системе $\left\{\chi_{k}\right\}_{k \in \mathbb{N}}(\kappa о э ф-$ фициенты $\hat{f}_{k}$ по системе функиий $\left\{\chi_{k}\right\}_{k \in \mathbb{N}}$ вычисляются так же, как в определении 1) имеет вид

$$
S_{n}(x)= \begin{cases}0, & \text { если не существует } \Delta_{i} \ni x, i \leqslant n, \\ \frac{1}{|\Delta|} \int_{\Delta} f d x, & \text { где } \Delta-\text { последний из } \Delta_{i} \ni x, i \leqslant n,\end{cases}
$$

и выполняется оченка $\sup _{n \in \mathbb{N}}\left|S_{n}(x)\right| \leqslant f^{m}(x)$, где $f^{m}(x)$ - точная верхняя грань $\left|\frac{1}{|\Delta|} \int_{\Delta} f d x\right|$ по всем отрезкам $\Delta(x \in \Delta \subset I)$, слабал максимальная функиия Харди-Литтлвуда. При этом рекурсивный ряд Фурье $f$ сходится $\kappa f$ во всех точках, где производная неопределенного интеграла равна $f$, т.е. почти всюду.

Кроме того, справедливы следующие утверждения.

(1) Для любой функиии $f \in L^{p}(I), 1<p<\infty$, рекурсивный ряд Фурье $f$ по системе $\left\{\chi_{k}\right\}_{k=1}^{\infty}$ сходится $\kappa f$ по метрике пространства Лебега $L^{p}(I)$.

(2) Если система $\Xi=\left\{\Delta_{k}\right\}_{k \in \mathbb{N}}$ покрывает промежуток I в смысле Витали так, что для любых $x \in I$ u $\varepsilon>0$ найдется один или два промежутка длины $<\varepsilon$, покрывающие точку $x$ вместе с некоторой ее окрестностью в $I$, то для любой непрерывной на I функиии $f$ ее рекурсивный ряд Фуръе по системе $\left\{\chi_{k}\right\}_{k=1}^{\infty}$ сходится $\kappa f$ равномерно на любом компакте из $I$.

(3) Пусть $\Xi_{n}=\left\{\Delta_{k} \in \Xi: k \leqslant n, \Delta_{k} \backslash \bigcup_{i=k+1}^{n} \Delta_{i} \neq \varnothing\right\}$ для любого $n \in \mathbb{N}$. Если найдется такое $M>0$, что для любой точки $x \in I$ и любого натурального $n$ число всех промежутков $\Delta_{k} \in \Xi_{n}$, содержащих точку $x$, не более, чем $M$, то для любой функиии $f \in L(I)$ рекурсивный ряд Фуръе $f$ по системе $\left\{\chi_{k}\right\}_{k=1}^{\infty}$ сходится $\kappa f$ по метрике пространства Лебега $L(I)$.

Часть приведенных утверждений обобщена на функции многих переменных в $[35,53]$.

Удобства использования орторекурсивных разложений можно показать в сравнении разложений по классической системе Хаара, являющейся простейшим примером ортогональных всплесков, и орторекурсивных разложений по системе характеристических функций двоичных отрезков.

Система Хаара обычно определяется на отрезке $[0,1]$ и состоит из следующих функций (cм. [11]).

Определение 2. Система Хаара - это система функций $X=\left\{\chi_{n}(x)\right\}_{n=1}^{\infty}, x \in[0,1]$, в которой $\chi_{1}(x)=\chi_{0}^{0}(x)=\equiv 1$, а функция $\chi_{n}(x)$ при $n=2^{k}+i, 1 \leqslant i \leqslant 2^{k}, k=0,1, \ldots$, определяется 
следующим образом:

$$
\chi_{n}(x)=\chi_{k}^{i}(x)=\left\{\begin{array}{cl}
0 & \text { при } x \notin\left[(i-1) 2^{-k}, i 2^{-k}\right], \\
2^{k / 2} & \text { при } x \notin\left((i-1) 2^{-k},(2 i-1) 2^{-k-1}\right), \\
-2^{k / 2} & \text { при } x \notin\left((2 i-1) 2^{-k-1}, i 2^{-k}\right) .
\end{array}\right.
$$

В концах отрезка $[0,1]$ функции Хаара непрерывны, а в точках разрыва регулярны, т.е. равны среднему арифметическому пределов слева и справа.

Система Хаара - полная ортонормированная система (см. [11]). Все функции Хаара $\chi_{n}(x)$, $n>2$, получаются двоичными сжатиями и сдвигами функции $\chi_{2}(x)$.

Теперь определим на отрезке $[0,1]$ другую неортогональную систему нормированных функций.

Определение 3. Система функций $\Lambda=\left\{\lambda_{n}(x)\right\}_{n=1}^{\infty}, x \in[0,1]$, состоит из функции $\lambda_{1}(x)=$ $\chi_{1}(x)=\equiv 1$, а функции $\lambda_{2 n-2}(x)$ и $\lambda_{2 n-1}(x)$ при $n=2^{k}+i, 1 \leqslant i \leqslant 2^{k}, k=0,1, \ldots$, определяются следующим образом:

$$
\begin{aligned}
& \lambda_{2 n-2}(x)=\sqrt{2} \chi_{n}^{+}(x)= \begin{cases}0 & \text { при } x \notin\left[(i-1) 2^{-k},(2 i-1) 2^{-k-1}\right], \\
2^{(k+1) / 2} & \text { при } x \in\left((i-1) 2^{-k},(2 i-1) 2^{-k-1}\right),\end{cases} \\
& \lambda_{2 n-1}(x)=\sqrt{2} \chi_{n}^{-}(x)= \begin{cases}0 & \text { при } x \notin\left[(2 i-1) 2^{-k-1}, i 2^{-k}\right], \\
2^{(k+1) / 2} & \text { при } x \in\left((2 i-1) 2^{-k-1}, i 2^{-k}\right) .\end{cases}
\end{aligned}
$$

Пусть в концах отрезка $[0,1]$ функции $\lambda_{2 n-2}(x)$ и $\lambda_{2 n-1}(x)$ непрерывны, а в точках разрыва регулярны, т.е. равны среднему арифметическому пределов слева и справа. Они являются с коэффициентом $\sqrt{2}$ положительной и отрицательной частью функции Хаара $\chi_{n}(x)$ (кроме точки $\left.(2 i-1) 2^{-k-1}\right), \chi_{n}(x)=\frac{1}{\sqrt{2}} \lambda_{2 n-2}(x)-\frac{1}{\sqrt{2}} \lambda_{2 n-1}(x), n>1, x \in[0,1]$.

Из вида частичных сумм $S_{N}^{\chi}(f, x)$ и $S_{N}^{\lambda}(f, x)$ рядов Фурье по системе $X$ Хаара (см. [11]) и вида частичных сумм орторекурсивного ряда Фурье по системе $\Lambda$ (см. теорему 5 ), следует, что

$$
S_{N}^{\chi}(f, x) \equiv S_{2 N-1}^{\lambda}(f, x)
$$

для любого $N \in \mathbb{N}$. Легко также проверить (например, используя указанное равенство частичных сумм), что коэффициенты Фурье орторекурсивного разложения $\hat{f}_{n}^{\lambda}$ связаны с коэффициентами Фурье-Хаара $\hat{f}_{n}^{\chi}$ и между собой следующим образом:

$$
\hat{f}_{1}^{\lambda}=\hat{f}_{1}^{\chi}, \quad \hat{f}_{2 n-2}^{\lambda}=-\hat{f}_{2 n-1}^{\lambda}=\sqrt{2} \hat{f}_{n}^{\chi}, \quad n>1 ; \quad \hat{f}_{n}^{\lambda}=\frac{1}{\sqrt{2}} \hat{f}_{2 n-1}^{\lambda}+\frac{1}{\sqrt{2}} \hat{f}_{2 n}^{\lambda}, \quad n \in \mathbb{N} .
$$

Верен и более общий факт, при заменах каких-либо функций Хаара парой соответствующих функций системы $\Lambda$ получится система функций, частичные суммы орторекурсивного разложения, в которые функции этих пар одновременно входят или не входят, имеют тот же вид, что и частичные суммы ряда Фурье по системе Хаара (см. $[4,37])$, где этот факт установлен для систем функций, среди которых имеется и система Хаара.

Особенностью орторекурсивных разложений по переполненным системам является возможность коррекции погрешностей, возникающих при вычислении коэффициентов разложения. Такая возможность отсутствует при разложении в ряды Фурье по ортогональным системам. Так при удалении из системы $\Lambda$ любого конечного числа функций частичные суммы по уменьшенной системе по теореме 5 начиная с некоторого номера будут иметь тот же вид, что и по самой системе $\Lambda$. Тот же факт будет верен и при совершении любого конечного числа ошибок в вычислении коэффициентов орторекурсивных разложений. Случай с бесконечным числом ошибок был изучен В. В. Галатенко (см. $[2,55])$.

Оказывается, что для нормированных систем, орторекурсивные разложения по которым абсолютно устойчивы к любому конечному числу ошибок, в случае таких относительных ошибок $\left\{\varepsilon_{n}\right\}_{n=1}^{\infty}$, что $\left|\varepsilon_{n}\right| \leqslant q<1, n=1,2,3, \ldots$, орторекурсивное разложение с ошибками всегда сходится к разлагаемому элементу.

Изучалась сходимость почти всюду орторекурсивных разложений в терминах множителей Вейля (см. $[5,6,56]$ Напомним определение. 
Определение 4. Неубывающая числовая последовательность $\left\{\lambda_{n}\right\}_{n=1}^{\infty} \subset[1, \infty)$ называется множителем Вейля сходимости почти всюду орторекурсивного разложения по последовательности $\left\{e_{n}(x)\right\}_{n=1}^{\infty} \subset L^{2}(\Omega)$, если из сходимости ряда $\sum_{n=1}^{\infty} \lambda_{n}\left|\hat{f}_{n}\right|^{2}$ следует сходимость почти всюду орторекурсивного разложения функции $f: \sum_{n=1}^{\infty} \hat{f}_{n} e_{n}(x)$.

Условием на числовую последовательность $\left\{\lambda_{n}\right\}_{n=1}^{\infty}$, необходимым и достаточным для того, чтобы эта последовательность была множителем Вейля сходимости почти всюду орторекурсивного разложения по каждой нормированной системе, является сходимость ряда

$$
\sum_{n=1}^{\infty} \frac{1}{\lambda_{n}}
$$

Это условие может быть существенно ослаблено, если ограничиться рассмотрением орторекурсивных разложений, сходящихся по норме к разлагаемой функции (что имеет место, в частности, для полных ортонормированных систем). Более конкретно, верна следующая теорема.

Теорема 6. Если орторекурсивное разложсение функиии $f(x) \in L^{2}(\Omega)$ по последовательности нормированных функиий $\left\{e_{n}(x)\right\}_{n=1}^{\infty}$ сходится $\kappa f(x)$ в $L^{2}(\Omega)$ и сходится ряд

$$
\sum_{n=1}^{\infty} \sqrt{n}\left|\hat{f}_{n}\right|^{2},
$$

то орторекурсивное разложение $\sum_{n=1}^{\infty} \hat{f}_{n} e_{n}(x)$ сходится $\kappa f(x)$ почти всюду на $\Omega$.

Таким образом, для орторекурсивных разложений, сходящихся по норме к разлагаемой функции, множителем Вейля является последовательность $\lambda_{n}=\sqrt{n}$.

Заметим, что для ортогональных разложений этот результат был получен А. Вейлем в 1909 г. Он неоднократно улучшался. Наилучший для всех ортонормированных систем результат о том, что последовательность $\lambda_{n}=\ln ^{2}(1+n)$ является множителем Вейля, был получен Д. Е. Меньшовым и Г. Радемахером в 1922-1923 гг. (см. [11, гл. 9, § 1]).

Ранее уже отмечалось, что для нормированных систем, орторекурсивные разложения по которым абсолютно устойчивы к любому конечному числу ошибок, в случае таких относительных ошибок $\left\{\varepsilon_{n}\right\}_{n=1}^{\infty}$, что $\left|\varepsilon_{n}\right| \leqslant q<1, n=1,2,3, \ldots$, орторекурсивное разложение с ошибками всегда сходится по норме к разлагаемому элементу. Для таких орторекурсивных разложений с ошибками верна также следующая теорема (см. [7]).

Теорема 7. Пусть система нормированных функиий $\left\{e_{n}(x)\right\}_{n=1}^{\infty}$ такова, что орторекурсивное разложсние по ним абсолютно устойчиво к любому конечному числу ошибок. Пусть орторекурсивное разложение функции $f(x) \in L^{2}(\Omega)$ совершено с такими относительными ошибками $\left\{\varepsilon_{n}\right\}_{n=1}^{\infty}$, ито $\left|\varepsilon_{n}\right| \leqslant q<1, n=1,2,3, \ldots$, и сходится ряд

$$
\sum_{n=1}^{\infty} \sqrt{n}\left|\hat{f}_{n}^{\mathrm{err}}\right|^{2}
$$

то такое орторекурсивное разложение $\sum_{n=1}^{\infty} \hat{f}_{n}^{\text {err }} e_{n}(x)$ сходится $\kappa f(x)$ почти всюду на $\Omega$.

В $[8,9,57]$ изложены результаты о сходимости почти всюду орторекурсивных разложений по системам специального вида.

Изучались орторекурсивные разложения Фурье-Стилтьеса (см. [30,33,34]), рекурсивные разложения мер (см. [25-29,31,32]). Обобщения орторекурсивных разложений в терминах операторов и систем элементов рассматривались А. Ю. Кудрявцевым, А. В. Политовым, В. В. Галатенко, Т. П. Лукашенко и В. А. Садовничим (см. $[4,19,20,36,37,46,49])$. 


\section{СПИСОК ЛИТЕРАТУРЫ}

1. Блаттер K. Вейвлет-анализ. Основы теории. - М.: Техносфера, 2004-2008.

2. Галатенко В. В. Об орторекурсивном разложении с ошибками в вычислении коэффициентов// Изв. РАН. Сер. мат. - 2005. - 69, № 1. - C. 3-16.

3. Галатенко В. В., Лукашенко Т. П., Садовничий В. А. Критерий совпадения орторекурсивных разложений по последовательностям подпространств// Воронеж. зимняя мат. школа «Современные методы теории функций и смежные проблемы» (Воронеж, 2013). - Воронеж: ВГУ, 2013. - С. 47-50.

4. Галатенко В. В., Лукашенко Т. П., Садовничий В. А. Орторекурсивные разложения// в кн.: Современные проблемы математики и механики/ K 80-летию мех.-мат. ф-та МГУ им. М. В. Ломоносова. - М.: Изд-во МГУ, 2014. - С. 13-25.

5. Галатенко В. В., Лукашенко Т. П., Садовничий В. А. О сходимости орторекурсивных разложений почти всюду// 18 Междунар. Саратов. зимняя школа «Современные проблемы теории функций и их приложения» (Саратов, 27 января - 3 февраля 2016). - Саратов: Научная книга, 2016. - C. 105-110.

6. Галатенко В. В., Лукашенко Т. П., Садовничий В. А. О множителях Вейля сходимости почти всюду орторекурсивных разложений// IX Междунар. симп. «Ряды Фурье и их приложения» (Ростов-наДону, 27 мая - 3 июня 2014). - Ростов-на-Дону, 2016. - С. 4-8.

7. Галатенко В. В., Лукашенко Т. П., Садовничий В. А. О множителях Вейля орторекурсивных разложений с ошибками в вычислении коэффициентов// Воронежская зимняя мат. школа «Современные методы теории функций и смежные проблемы» (Воронеж, 26 января - 1 февраля 2017). - Воронеж: ВГУ, 2017. - С. 77-79.

8. Галатенко В. В., Лукашенко Т. П., Садовничий В. А. О сходимости почти всюду орторекурсиввных разложений по системам сжатий и сдвигов// 13 Междунар. Казанск. летняя школа-конф. «Теория функций, ее приложения и смежные вопросы» (Казань, 21-27 августа 2017). - Казань: Мат. центр им. Н. И. Лобачевского, 2017. - 54. - С. 99-101.

9. Галатенко В. В., Лукашенко Т. П., Садовничий В. А. Сходимость почти всюду орторекурсиввных разложений по функциональным системам специального вида// 19 Междунар. Саратов. зимняя школа «Современные проблемы теории функций и их приложения», посв. 90-летию со дня рождения акад. П. Л. Ульянова (Саратов, 29 января - 2 февраля 2018). - Саратов: Научная книга, 2018. - С. 101-104.

10. Добеши И. Десять лекций по вейвлетам. - М.-Ижевск: Регулярная и хаотическая динамика, 2001.

11. Кашин Б. С., Сааклн А. А. Ортогональные ряды. - М.: АФЦ, 1999.

12. Кудрявцев А. Ю. Орторекурсивные разложения по системам сжатий и сдвигов// Междунар. школасемин. по геом. и анализу, посв. 90-летию Н. В. Ефимова (Абрау-Дюрсо, 5-11 сентября 2000). Ростов-на-Дону, 2000. - С. 127-129.

13. Кудрявиев А. Ю. Орторекурсивные разложения по системам сжатий и сдвигов фиксированной функции// Воронеж. зимняя мат. школа «Современные методы теории функций и смежные проблемы» (27 января - 4 февраля 2001). - Воронеж: ВГУ, 2001. - С. 161-162.

14. Кудрявцев А. Ю. Орторекурсивные разложения по системам сжатий и сдвигов фиксированной функции// Междунар. школа-семин. по геом. и анализу, посв. памяти Н. В. Ефимова (Абрау-Дюрсо, 5-11 сентября 2002). - Ростов-на-Дону, 2002. - С. 133-135.

15. Кудрявцев А. Ю., Лукашенко Т. П. Орторекурсивные разложения по системам сжатий и сдвигов// II Междунар. конф. «Оптимизация конечно-элементных приближений, сплайны и всплески» (СПб., 25-29 июня 2001). - СПб.: НИИХ СПбГУ, 2001. - С. 141-143.

16. Кудрявиев А. Ю. Орторекурсивные разложения по системам сжатий и сдвигов// 11 Саратов. зимняя школа «Современные проблемы теории функций и их приложения», посв. памяти Н. К. Бари и Д. Е. Меньшова (Саратов, 28 января - 4 февраля 2002). - Саратов: СГУ, 2002. - С. 106-108.

17. Кудрявиев А. Ю. Орторекурсивные разложения по системам неортогональных всплесков// Воронеж. зимняя мат. школа «Современные методы теории функций и смежные проблемы» (Воронеж, 26 января - 2 февраля 2003). - Воронеж: ВГУ, 2003. - С. 137-138.

18. Кудрявиев А. Ю. Орторекурсивные разложения по неортогональным всплескам// Воронеж. зимняя мат. школа «Современные методы теории функций и смежные проблемы» (Воронеж, 2011). - Воронеж: ВГУ, 2011. - С. 189-191.

19. Кудрявцев А. Ю. О сходимости орторекурсивных разложений по неортогональным всплескам// Мат. заметки. - 2012. - 92, № 5. - С. 707-720.

20. Кудрлвиев А. Ю. О скорости сходимости орторекурсивных разложений по неортогональным всплескам// Изв. РАН. Сер. мат. - 2012. - 76, № 4. - С. 49-64. 
21. Кудрявцев А. Ю. Орторекурсивные разложения по неортогональным всплескам/ Дисс. на соиск. уч. степ. канд. физ.-мат. наук, 2013.

22. Лукашенко Т. П. Рекурсивные разложения, подобные ортогональным// VII Междунар. конф. «Ряды Фурье и их приложения» (Ростов-на-Дону, 26 мая - 1 июня 1999). - Ростов-на-Дону: РГЭА, 1999. C. 331 .

23. Лукашенко Т. П. Об орторекурсивных разложениях по характеристическим функциям промежутков// Школа-конф. «Теория функций, ее приложения и смежные вопросы», посв. 130-летию со дня рожд. Д. Ф. Егорова. - Казань: Казанск. мат. об-во, 1999. - С. 142-143.

24. Лукашенко Т. П. О свойствах орторекурсивных разложений по неортогональным системам// Вестн. Моск. ун-та. Сер. 1. Мат. Мех. - 2001. - № 1. - С. 6-10.

25. Лукашенко Т. П. О рекурсивных разложениях функций и мер// 15 Саратов. зимняя школа «Современные проблемы теории функций и их приложения», посв. 125-летию со дня рождения В. В. Голубева и 100-летию СГУ (Саратов, 27 января - 3 февраля 2010). - Саратов: Изд-во Саратов. ун-та, 2010. C. $102-105$.

26. Лукашенко Т. П. О рекурсивных разложениях мер// Междунар. науч. конф. «Современные проблемы анализа и преподавания математики», посв. 105-летию акад. С. М. Никольского (Москва, 17-19 мая 2010). - M., 2010. - C. 27.

27. Лукашенко Т. П. Рекурсивные разложения мер// VI Междунар. симп. «Ряды Фурье и их приложения» (Ростов-на-Дону, 28 мая - 1 июня 2010). - Ростов-на-Дону, 2010. - С. 19.

28. Лукашенко Т. П. Рекурсивные разложения мер// Междунар. конф. «Метрическая геометрия поверхностей и многогранников», посв. 100-летию со дня рожд. Н. В. Ефимова (Москва, 18-21 августа 2010). - М.: Макс Пресс, 2010. - С. 89.

29. Лукашенко Т. П. О сходимости рекурсивных разложений мер// Воронеж. зимняя мат. школа «Современные методы теории функций и смежные проблемы» (Воронеж, 2011). - Воронеж: ВГУ, 2011. - C. 203-204.

30. Лукашенко Т. П. Рекурсивные разложения Фурье-Стилтьеса// 10 Междунар. Казанск. летняя науч. школа-конф. «Теория функций, ее приложения и смежные вопросы» (Казань, 1-7 июля 2011), 2011. - 43. - C. 233-238.

31. Лукашенко Т. П. О свойствах рекурсивных разложений мер// в кн.: Современные проблемы математики и механики/ К 100-летию со дня рожд. Н. В. Ефимова. - М.: Изд-во МГУ, 2011. - С. 90-96.

32. Лукашенко Т. П. О рекурсивных разложениях мер// Мат. заметки. - 2012. - 91, № 5. - С. 720-729.

33. Лукашенко Т. П. Рекурсивные ряды Фурье-Стилтьеса// 16 Саратов. зимняя школа «Современные проблемы теории функций и их приложения» (Саратов, 27 января - 3 февраля 2012). - Саратов: Научная книга, 2012. - С. 108-109.

34. Лукашенко Т. П. Орторекурсивные разложения Фурье-Стилтьеса// Тр. Мат. ин-та РАН им. В. А. Стеклова. - 2013. - 280. - С. 1-7.

35. Лукашенко Т. П., Белоусов К. В. О некоторых свойствах орторекурсивных разложений функций многих переменных по системе характристических функций брусов// в кн.: Современные проблемы математики и механики/ K 105-летию С. М. Никольского. - М.: Изд-во МГУ, 2011. - С. 52-60.

36. Лукашенко Т. П., Садовничий В. А. О рекурсивных разложениях по цепочке систем// Докл. РАН. 2009. - 425, № 6. - С. $741-746$.

37. Лукашенко Т. П., Садовничий В. А. Орторекурсивные разложения по подпространствам// Докл. PAH. - 2012. - 445, № 2. - C. 135-138.

38. Лукашенко Т. П., Сквориов В. А., Солодов А. П. Обобщенные интегралы. - М.: Либроком, 2009.

39. Малла С. Вейвлеты в обработке сигналов. - М.: Мир, 2005.

40. Натансон И. П. Теория функций вещественной переменной. - СПб.: Лань, 1999.

41. Новиков И. Я., Протасов В. Ю., Скопина М. А. Теория всплесков. - М.: Физматлит, 2005.

42. Политов A. В. Орторекурсивные разложения в гильбертовых пространствах// 14 Саратов. зимняя школа «Современные проблемы теории функций и их приложения», посв. памяти акад. П. Л. Ульянова (Саратов, 28 января - 4 февраля 2008). - Саратов: СГУ, 2008. - С. 146-147.

43. Политов A. В. Орторекурсивные разложения по системе сжатий и сдвигов нескольких функций// Воронеж. зимняя мат. школа «Современные методы теории функций и смежные проблемы» (Воронеж, 2009). - Воронеж: ВГУ, 2009. - С. 144-145.

44. Политов A. В. Орторекурсивные разложения по системе сжатий и сдвигов нескольких функций// Междунар. конф. «Современные проблемы математики, механики и их приложения», посв. 70-летию 
ректора МГУ акад. В. А. Садовничего. - М.: Университетская книга, 2009. - С. 89-90.

45. Политов А. В. Критерий сходимости орторекурсивных разложений в гильбертовых пространствах// 15 Саратов. зимняя школа «Современные проблемы теории функций и их приложения», посв. 125летию со дня рожд. В. В. Голубева и 100-летию СГУ (Саратов, 27 января - 3 февраля 2010). Саратов: Изд-во Саратов. ун-та, 2010. - С. 141-142.

46. Политов A. В. Орторекурсивные разложения в гильбертовых пространствах// Вестн. Моск. ун-та. Сер. 1. Мат., Мех. - 2010. - № 3. - С. 3-7.

47. Политов А. В. Критерий сходимости орторекурсивных разложений в гильбертовых пространствах// Воронеж. зимняя мат. школа «Современные методы теории функций и смежные проблемы» (Воронеж, 2011). - Воронеж: ВГУ, 2011. - С. 268-269.

48. Политов А. В. Достаточные условия сходимости орторекурсивных разложений на квадрате// 16 Саратов. зимняя школа «Современные проблемы теории функций и их приложения» (Саратов, 27 января - 3 февраля 2012). - Саратов: Научная книга, 2012. - С. 135.

49. Политов А. В. Критерий сходимости орторекурсивных разложений в евклидовых пространствах// Мат. заметки. - 2013. - 93, № 4. - С. 637-640.

50. Политов A. В. Условия сходимости орторекурсивных разложений в гильбертовых пространствах/ Дисс. на соиск. уч. степ. канд. физ.-мат. наук - М.: МГУ им. М. В. Ломоносова, 2013.

51. Сакс С. Теория интеграла. - М.: ИЛ, 1949.

52. Фрейзер M. Введение в вейвлеты в свете линейной алгебры. - М.: БИНОМ-Лаборатория знаний, 2008.

53. Фуфаев Д. В. Промежуточный случай регулярности в задаче дифференцирования кратных интегралов// Изв. Саратов. ун-та. Сер. Мат. Мех. Информ. - 2014. - 14, № 4. - С. 401-407.

54. Чуи K. Введение в вейвлеты. - М.: Мир, 2001.

55. Galatenko V. V., Lukashenko T. P., Sadovnichiy V. A. The absolute stability of orthorecursive expansions in redundant systems of subspaces// in: Continuous and Distributed Systems. II. Theory and Applications/ Studies in Systems, Decision and Control. - Springer, 2015. - 30. - P. 3-10.

56. Galatenko V. V., Lukashenko T. P., Sadovnichiy V. A. Convergence almost everywhere of orthorecursive expansions in functional systems// in: Advances in Dynamical Systems and Control/ Studies in Systems, Decision and Control. - Springer, 2016. - 69. - P. 3-11.

57. Galatenko V. V., Lukashenko T. P., Sadovnichiy V. A. Convergence almost everywhere of orthorecursive expansions in systems of translates and dilates// in: Fundamentals of Modern Mathematics and Mechanics. - Springer, 2018. - P. 3-11.

58. De Vore R. A., Temlyakov V. N. Some remarks on greedy algorithms// Adv. Comput. Math. — 1996. 5. - P. 173-187.

59. Meyer Y. Ondelettes et opérateurs. - Paris: Hermann, 1990-1991.

Галатенко Владимир Владимирович

Московский государственный университет им. М. В. Ломоносова

E-mail: vgalat@imscs.msu.ru

Лукашенко Тарас Павлович

Московский государственный университет им. М. В. Ломоносова

E-mail: lukashenko@mail.ru

Садовничий Виктор Антонович

Московский государственный университет им. М. В. Ломоносова

E-mail: info@rector.msu.ru 